\title{
Repeatable Extrinsic Stereo Camera Calibration by Moving Chessboard in a Straight-line
}

\author{
Megha Simlote \\ Assistant System Engineer, Tata \\ Consultancy Services, Bangalore
}

\author{
Pratibha Agrawal \\ Assistant System Engineer, Tata \\ Consultancy Services, Bangalore
}

\author{
Sunil Pathak \\ Amity School of Engineering \& \\ Technology, Department of \\ computer Engineering \\ Amity University, Jaipur, Rajasthan
}

\begin{abstract}
Camera calibration is the procedure of identifying camera parameters for rectifying and un-distorting the images. In this paper Calibration of Stereo Camera using the set of images taken by 2 cameras (from left and right direction) and study about the stability as well as statically analysis of parameters is carried out by physically changes the angle of camera. For estimating extrinsic parameters the images could be taken in a way, covering whole surface area of the camera sensor i.e. at different depth and orientation but later, it was observed that if the images taken were in a straight line at different depths covering the same area of camera sensor then the repeatability could be seen in the results of extrinsic parameters.
\end{abstract}

\section{Keywords}

2D Image, 3D Image, Camera Calibration, Chessboard, angle.

\section{INTRODUCTION}

Camera Calibration is a necessary step to extract metric information from 2D images. The goal is to recover the mapping between the 3D space $[9,20]$ and 2D image. Camera calibration is an important issue in the field of computer vision. This can be separated in to two sets of transformation. First is mapping of the $3 \mathrm{D}$ points in the scene to $3 \mathrm{D}$ coordinates in camera frame, described by extrinsic parameters. Second is mapping of the $3 \mathrm{D}$ points in the camera frame to the 2D coordinates in the image plane, described by intrinsic parameters, which provides the geometry and optical features of the camera. These 2 transformations can be expressed by the ideal pinhole camera model $[6,7]$. Finding out these parameters is known as calibration.

\section{$\mathrm{X}=(\mathrm{X}, \mathrm{Y}, \mathrm{Z}, 1) \mathrm{T}$ - coordinate of point in 3-D world}

$\mathrm{Xcam}=[\mathrm{R} t] \mathrm{X}-3-\mathrm{D}$ coordinate of same point in camera frame. Where, $\mathrm{R}-3 \mathrm{x} 3$ rotation matrix, $\mathrm{t}-3 \mathrm{x} 1$ translation matrix and Image coordinate of that 3-D point: $\mathrm{X}=(\mathrm{x}, \mathrm{y}, 1) \mathrm{T}$.

3-D to 2-D mapping becomes:

$\mathrm{x}=\mathrm{K}[\mathrm{R} \mathrm{t}] \mathrm{X}$, Where, $\mathrm{K}-$ it is camera matrix which contains intrinsic parameter

$\mathrm{K}=\left[\left[\begin{array}{lll}f x & 0 & c x\end{array}\right],\left[\begin{array}{lll}0 & f y & c y\end{array}\right],\left[\begin{array}{lll}0 & 0 & 1\end{array}\right]\right]$

Where, fx and fy are focal length, cx and cy are coordinates of the principal point, $\mathrm{R}$ and $\mathrm{t}$ constitutes extrinsic parameter of camera. Finding out these parameters is known as calibration.

There are 2 approaches for camera calibration first is Metric calibration which resolves the camera model with the help of metric information of a reference object. It is performed by observing a calibration object whose geometry dimensions are known with high precision. In $[1,2,3]$ calibration using rotation, with analysis of sources of error is calculated and In [4, 5], Calibration Accuracy Assessment Methods for Stereovision Sensors Used in Vehicles. Calibration object is a 3D object with several planes orthogonal to each others; sometimes a 2D plane undergoing a known translation or free movement is utilized. Another type of calibration method is Self-calibration which does not use metric information from the scene or any object. Metric calibration method provides better results than Self calibration [6].

A calibration pattern needs to be prepared though the setup is very easy and only a planar object is attached with a chessboard is used. Pattern shall cover around $25 \%$ of width and height of field of view. Entire field of view shall be covered specifically corners while taking images. Multiple angles of chessboard $[10,11,12,13]$ and entire depth shall be covered $[15,17]$. Our current experiment is concerned with the extrinsic parameters that are 3 translation vectors and 3 rotation vectors. From this paper, we want to raise the issue that we observed rather covering whole surface area of sensor one should capture images covering a single area of sensor at different depths to get the repeatability in extrinsic results.

\section{OVERVIEW OF METHOD USED}

When extrinsic parameters were estimated by using the images covering whole surface area of camera sensor i.e. taking images at different depth and orientations then it is found that there is no repeatability in results. Hence, by using the images taken in a straight line covering same region of camera sensor it is observed that extrinsic results are repeatable. For intrinsic parameters the images covering whole surface area of camera sensor are good enough for estimating them $[2,9,18,19]$

\section{PROPOSED PROCEDURE OF CAMERA CALIBRATION ALGORITHM}

In procedure of camera calibration, intrinsic parameters are estimated by taking the chessboard images covering the whole surface area of camera sensor i.e. at every orientation and different depths. For this estimation 2-3 set of images were taken containing about 50 pairs of images (for left and right camera) each. The baseline between two stereo cameras is 20 cm. [4]

Step-I Calculated intrinsic parameters using OpenCV library [8]

cv2.findChessboardCorners(): It finds the pattern in chess board, returns the corners of chessboard. 
1. cv2.cornerSubPix(): Increase accuracy in image points.

2. cv2.drawChessboardCorners(): Draws pattern on the chessboard.

3. cv2.calibrateCamera(): Returns camera matrix and distortion coefficients.

Step-II Calculate Extrinsic Parameters from same set of images

cv2.stereoCalibrate() function is used to calculate extrinsic parameter of camera by using output of Step-I of Intrinsic Parameters.

- Rotation matrix could be converted to rotation vector with the help of cv2.Rodrigues (rotationmatrix ,rotationvector)function which returns rotation vector [8].

- We found no repeatability in the extrinsic parameter results while there is no change in the angle of camera.

Step-III

- Calculate a fixed set of intrinsic parameter, which are required to calculate extrinsic parameters by using images covering whole surface area at different orientations, depth and as well as the images using flat surface of chessboard with an error of $1.55 \%$ and $1.22 \%$ respectively. (refer table1(a) and table1(b)).

- After fixing the set of intrinsic (as the camera used is same) another set of images were taken with a change of 0.1 degree in angle of camera and extrinsic parameters were calculated using previous set of intrinsic parameter but this time the results does not reflects the change of 0.1 degree in pan angle with the average error of $39.2 \%$ (refer table2).

- $\quad$ Again the 5 set each contains 26 pairs of images were taken at different depth but in a straight line in both positions first chessboard up then down as shown in fig1 hence, using the same region of sensors without changing the angle of camera. When the extrinsic parameters were estimated for these set of images using those fixed intrinsic we found repeatability in the results with the average error of $0.27 \%$ and $0.25 \%$ respectively. (refer table3(a) and table3(b)).

- With the change of 0.1 degree in camera angle, four set of 22 pairs of images were taken at different depth and in a straight line using same region of camera sensor, the extrinsic parameters reflect the change of 0.1 degree in the pan angle calculated through calibration process with the average error of $11 \%$ (refer table4).

- At last, the 6 set of 22 pairs of images were taken with the change of 0.2 degree in camera angle and again the results reflected change of 0.2 degree in pan angle of extrinsic parameters with the average error of $9.9 \%$ (refer table5) $[1,3,10]$

\section{Step-IV End}

\section{IMPLEMENTATION SETUP AND SIMULATION RESULTS}

Implementation is carried out using python 3.7 [8]. Source code for implementing camera calibration is written by using python 3.7 which is as below.

From the simulation result it is cleared that extrinsic parameters with no change in angle of camera for whole surface area and Extrinsic with no change in angle of camera for whole surface area with flat surface and re-projection error is given in table 1(a) and 1(b).

Table 1(a): Extrinsic with no change in angle of camera (whole surface area)

\begin{tabular}{|c|c|c|c|}
\hline $\begin{array}{c}\text { Experiment } \\
\text { no. }\end{array}$ & Pan angle & Difference & $\begin{array}{c}\text { Re-projection } \\
\text { error }\end{array}$ \\
\hline $\mathbf{1 .}$ & 0.0087 & & 0.6680 \\
\hline $\mathbf{2 .}$ & 0.0310 & 0.0223 & 0.7148 \\
\hline $\mathbf{3 .}$ & 0.0181 & 0.0129 & 0.8198 \\
\hline $\mathbf{4 .}$ & -0.0084 & 0.0265 & 0.6470 \\
\hline $\mathbf{5 .}$ & -0.0108 & 0.0024 & 0.6652 \\
\hline
\end{tabular}

Table 1(b): Extrinsic with no change in angle of camera (whole surface area with flat surface)

\begin{tabular}{cccc}
$\begin{array}{c}\text { Experiment } \\
\text { no. }\end{array}$ & Pan angle & Difference & $\begin{array}{c}\text { Re-projection } \\
\text { error }\end{array}$ \\
\hline 1. & -0.0033 & & 0.5514 \\
\hline 2. & -0.0172 & 0.0139 & 0.5206 \\
\hline 3. & -0.00009 & 0.0171 & 0.4509 \\
\hline 4. & -0.0078 & 0.0077 & 0.4799 \\
\hline $\mathbf{5}$ & -0.0047 & 0.0125 & 0.4576 \\
\hline & & & \\
\hline
\end{tabular}

After that, fixing the set of intrinsic (as the camera used is same) another set of images were taken and extrinsic parameters were calculated using previous set of intrinsic parameter but this time the results does not reflects the change of 0.1 degree in pan angle with the error of $39.2 \%$.

Table 2: Extrinsic with 0.1-degree change in angle of camera (whole surface area)

\begin{tabular}{ccccc}
\hline $\begin{array}{c}\text { Experiment } \\
\text { no. }\end{array}$ & $\begin{array}{c}\text { Pan } \\
\text { angle }\end{array}$ & Difference & Error & $\begin{array}{c}\text { Re- } \\
\text { projection } \\
\text { error }\end{array}$ \\
\hline 1. & -0.5967 & & & 0.7396 \\
\hline 2. & -0.7658 & 0.1691 & $69 \%$ & 0.8008 \\
\hline 3. & -0.8294 & 0.0635 & $36.5 \%$ & 0.6219 \\
\hline 4. & -0.9257 & 0.096 & $4 \%$ & 0.6718 \\
\hline 5. & -1.1103 & 0.1846 & $84.6 \%$ & 0.7441 \\
\hline 6. & -1.0050 & 0.105 & $5 \%$ & 1.6603
\end{tabular}


Now, again the 5 set each contains 26 pairs of images were taken at different depth but in a straight line in both positions first chessboard up then down as shown in fig1 hence, using the same region of sensors without changing the angle of camera. When the extrinsic parameters were estimated for these set of images using those fixed intrinsic we found repeatability in the results with the average error of $0.27 \%$ and $0.25 \%$ respectively. (Refer table 3 (a) and table 3 (b)).

Table 3(a): Extrinsic with no change in angle of camera (same area when chessboard is up)

\begin{tabular}{cccc}
$\begin{array}{c}\text { Experiment } \\
\text { no. }\end{array}$ & Pan angle & Difference & $\begin{array}{c}\text { Re- } \\
\text { projection } \\
\text { error }\end{array}$ \\
1. & -0.0314 & & 0.3143 \\
\hline 2. & -0.0314 & 0.0 & 0.3481 \\
\hline 3. & -0.0346 & 0.0032 & 0.3142 \\
\hline 4. & -0.0303 & 0.0043 & 0.3145 \\
\hline 5. & -0.03517 & 0.0048 & 0.3235 \\
\hline
\end{tabular}

Table 3(b): Extrinsic with no change in angle of camera (same area when chessboard is down)

\begin{tabular}{cccc}
$\begin{array}{c}\text { Experiment } \\
\text { no. }\end{array}$ & $\begin{array}{c}\text { Pan } \\
\text { angle }\end{array}$ & Difference & $\begin{array}{c}\text { Re- } \\
\text { projection } \\
\text { error }\end{array}$ \\
\hline 1. & -0.0116 & & 0.1390 \\
\hline 2. & -0.0083 & 0.0033 & 0.1391 \\
\hline 3. & -0.0082 & 0.0001 & 0.1376 \\
\hline 4. & -0.0120 & 0.0038 & 0.1412 \\
\hline 5. & -0.0080 & 0.0040 & 0.1405 \\
\hline
\end{tabular}

With the change of 0.1 degree in camera angle, four set of images were taken at different depth and in a straight line using same region of camera sensor, the extrinsic parameters reflect the change of 0.1 degree in the pan angle with the error of $11 \%$.

Table 4: Extrinsic with 0.1-degree change in angle of camera (same area)

\begin{tabular}{ccc|cc}
\hline $\begin{array}{c}\text { Experim } \\
\text { ent no. }\end{array}$ & $\begin{array}{c}\text { Pan } \\
\text { angle }\end{array}$ & $\begin{array}{c}\text { Differenc } \\
\mathbf{e}\end{array}$ & $\begin{array}{c}\text { Erro } \\
\mathbf{r}\end{array}$ & $\begin{array}{c}\text { Re- } \\
\text { projection } \\
\text { error }\end{array}$ \\
\hline 1. & -1.5494 & & & 0.2269 \\
\hline $\mathbf{2 .}$ & -1.6337 & 0.084 & $16 \%$ & 0.2355 \\
\hline 3. & -1.7232 & 0.089 & $11 \%$ & 0.2362 \\
\hline 4. & -1.8181 & 0.094 & $6 \%$ & 0.2297 \\
\hline
\end{tabular}

At last, the images were taken with the change of 0.2 degree in camera angle and again the results reflected change of 0.2 degree in pan angle of extrinsic parameters with the error of $9.9 \%$.
Table 5: Extrinsic with 0.2-degree change in angle of camera (same area)

\begin{tabular}{ccccc}
\hline $\begin{array}{c}\text { Experimen } \\
\text { t no. }\end{array}$ & $\begin{array}{c}\text { Pan } \\
\text { angle }\end{array}$ & $\begin{array}{c}\text { Differenc } \\
\text { e }\end{array}$ & $\begin{array}{c}\text { Erro } \\
\mathbf{r}\end{array}$ & $\begin{array}{c}\text { Re- } \\
\text { projectio } \\
\text { n error }\end{array}$ \\
\hline 1. & -0.099 & & & 0.2580 \\
\hline $\mathbf{2 .}$ & -0.286 & 0.187 & $6.5 \%$ & 0.2814 \\
\hline 3. & -0.468 & 0.182 & $9 \%$ & 0.3382 \\
\hline $\mathbf{4 .}$ & -0.634 & 0.166 & $17 \%$ & 0.3197 \\
\hline $\mathbf{5 .}$ & -0.823 & 0.189 & $5.5 \%$ & 0.3301 \\
\hline 6. & - & & 11.5 & 0.3479 \\
& 2 & & & \\
\hline & & & &
\end{tabular}

\section{CONCLUSION AND FUTURE WORKS}

Initially, the intrinsic and extrinsic calibration data is estimated using the images provided and then the parameters are analyzed statistically and found that the data is not normally distributed, empirical distribution i.e. kernel density estimation is also tried but best fit obtained is beta distribution. It is found that when extrinsic parameters were estimated using whole surface area of camera sensor then the results are not repeating but when we tried them using the images taken at different depth and in straight line covering the same region of sensors the results seem to be repeatable while intrinsic estimation is done using the whole surface area of camera sensor.

We observed that, the Intrinsic parameters can be estimated using images covering whole surface area of camera sensor while extrinsic parameters can be found by taking images in a straight line covering same region of camera sensor and keeping object at different orientation and depth will provide the repeatability in the results of extrinsic with the change in the angle of camera and thus we get the new method of camera calibration using which we can observe the change in pan angle as the angle of camera changed. In future this research can be further used to extract data from the object placed at very far distance from the camera approximately in the range of $1 \mathrm{~km}$.

\section{REFERENCES}

[1] Effects of Camera Alignment Errors on Stereoscopic Depth Estimates, 1996 by Wenyi Zhao , N. Nandhakumar Pattern Recognition Volume 29, Issue 12, December 1996, Pages 2115-2126

[2] G.P.Stein, "Accurate internal camera calibration using rotation, with analysis of sources of error," in Proceedings of the 5th International Conference on Computer Vision (ICCV '95), pp. 230236,Cambridge,Mass,USA,June1995.

[3] Error Analysis of Calibration Parameters Estimation for Binocular Stereo Vision System ,2003 by Sergiu Nedevschi, Tiberiu Marita, Radu Danescu, Florin Oniga, Dan Frentiu, Ciprian Pocol microCAD International Scientific Conference, March, Miskolc, Hungary, pp. 5156

[4] Camera Calibration Method for Far Range Stereovision 
Sensors Used in Vehicles, by T. Marita ; F. Oniga ; S. Nedevschi ; T. Graf ; R. Schmidt, Intelligent Vehicles Symposium, 2006 IEEE Intelligent Vehicles Symposium

[5] Calibration Accuracy Assessment Methods for Stereovision Sensors Used in Vehicles, 2007 by Tiberiu Marita ; Florin Oniga ; Sergiu Nedevschi ; Thorsten Graf,Intelligent Computer Communication and Processing, 2007 IEEE International Conference on Intelligent Computer Communication and Processing

[6] How accurate can a stereovision measurement be ?, 2014 by Kristiaan Schreve, Research and Education in Mechatronics (REM), 2014 15th International Workshop Research and Education in Mechatronics (REM)

[7] A Flexible Online Camera Calibration Using Line Segments, 2015 by Yueqiang Zhang,1,2 Langming Zhou,1,2 Haibo Liu,1,2 and Yang Shang1,2,Journal of sensors.

[8] https://pypi.org/project/opencv-python

[9] Using vanishing points for camera calibration and coarse 3D reconstruction from a single image (E.Guillou, D.Meneveaux, E.Maisel, K.Bouatouch ),IRISA, Campus de Beaulieu, 35042 Rennes cedex, France

[10] F. C. Wu, Z. Y. Hu, and H. J. Zhu, "Camera calibration with moving one-dimensional objects," Pattern Recognition, vol.38, no.5, pp.755-765,2005.

[11] Z. Zhang, "A Flexible New Technique for Camera Calibration," IEEE Trans. Pattern Analysis and Machine Intelligence, vol. 22, no. 11, pp. 1330-1334, Nov. 2000

[12] A. Heyden and M. Pollefeys, "Multiple View Geometry," Emerging Topics in Computer Vision, G. Medioni and S.B. Kang, eds., chapter 3, pp. 45-108, Prentice Hall, 2003.
[13] Douskos, V.; Kalisperakis, I.; Karras, G.; Petsa, E. Fully automatic camera calibration using regular planar patterns. Int. Arch. Photogram. Remote Sens. Spatial Inf. Sci. 2008, 37, 21-26

[14] Kwon, H., Park, J., and Kak, A. C. (2007). A New Approach for Active Stereo Camera Calibration. In Proceedings 2007 IEEE International Conference on Robotics and Automation, pages 3180-3185. IEEE.

[15] Lhuillier, M. (2008). Automatic scene structure and camera motion using a catadioptric system. Computer Vision and Image Understanding, 109(2):186-203.

[16] Lim, J., Barnes, N., and Li, H. (2010). Estimating relative camera motion from the antipodal-epipolar constraint. IEEE transactions on pattern analysis and machine intelligence, 32(10):1907-1914.

[17] J. Lim, N. Barnes, and H. Li, "Estimating relative camera motion from the antipodal-epipolar constraint," IEEE Transactions on Pattern Analysis and Machine Intelligence, vol. 32, no. 10, pp. 1907-1914, 2010.

[18] M. Lhuillier, "Automatic scene structure and camera motion using a catadioptric system," Computer Vision and Image Understanding, vol. 109, no. 2, pp. 186-203, 2008.

[19] J. Chen, K. Benzeroual, and R. S. Allison, "Calibration for high-definition camera rigs with marker chessboard," in Proceedings of the 2012 IEEE Computer Society Conference on Computer Vision and Pattern Recognition Workshops (CVPRW '12), pp. 29-36, June 2012.

[20] J. Chen, K. Benzeroual, and R. S. Allison, "Robust homography for real-time image un-distortion," in Proceedings of the 2013 International Conference on 3D Imaging (IC3D '13), pp. 1-8, December 2013. 